\title{
Solving Multi Objective Linear Programming Problems Using Intuitionistic Fuzzy Optimization Method: A Comparative Study
}

\author{
S. K. Bharatiand and S. R. Singh
}

\begin{abstract}
The paper aims to give computational algorithm to solve a multi objective linear programming problem using intuitionistic fuzzy optimization method. It also includes some basic properties of intuitionistic fuzzy set and operations on it. The development of algorithm is based on principle of optimal decision set obtained by intersection of various intuitionistic fuzzy decision sets which are obtained corresponding to each objective function. Further, as the intuitionistic fuzzy optimization technique utilizes degree of belonging and degree of non-belonging, we made a comparative study of linear and nonlinear membership function for belonging and nonbelonging to see its impact on optimization and to get insight in such optimization process. The developed algorithm has been illustrated by a numerical example.
\end{abstract}

Index Terms - Intuitionistic fuzzy set, multi objective linear programming, membership function, non-membership function.

\section{INTRODUCTION}

In several optimization problems, it has been observed that a small violation in given constraints or conditions may lead to more efficient solution to the problem. Such situations appear in frequent way in real life modeling, as a matter of fact in optimization problems; many times it is not practical to fix accurate parameters as many of these are obtained through approximation or through some kind of human observation. For example in a production optimization problem, it is not necessary that all the produced are of good quality and are completely sellable on a fixed price. There is possibility that some of the products may be defective and are not sellable on the fixed price. Further prices of raw material as well as market price of finished product may vary depending on its surplus/deficiency in the market due to some uncontrollable situations. Thus it is evident that prices and/or productions are not purely deterministic but in general these are imprecise or nondeterministic and thus such problems of optimization are to be dealt with help of some non-classical methods.

Modeling of most of real life problems involving optimization process turns out to be multi objective programming problem in a natural way. Such multi objective programming problems may in general comprise of conflicting objectives. For example, if we consider a

Manuscript received August 9, 2013; revised October 13, 2013.

The authors are with the Department of Mathematics, Banaras Hindu University, Varanasi- 221005, India (e-mail: singh_shivaraj@rediffmail.com, skmaths.bhu@gmail.com). problem of agricultural production planning, the optimal model should have the objectives of maximizing the profit and minimizing the inputs and cost of cultivation. Thus these objectives are conflicting in nature and hence solution of such problems are in general compromise solutions which satisfy each objective function to a degree of satisfaction and a concept of belonging and non-belonging arises in such situations. It was Zimmermann [1], [2] who first used the fuzzy set introduced by Zadeh [3] for solving the fuzzy multi objective mathematical programming problem. Optimization in fuzzy environment was further studied and was applied in various areas by many researchers such as Tanaka [4], Luhandjula [5], Sakawa[6] etc. A brief review of studies of various research workers on optimization under uncertainty can be found in work of Sahinidis [7].

In view of growing use of fuzzy set in modeling of problems under situations when information available is imprecise, vague or uncertain, various extension of fuzzy sets immerged. In such extensions, Atanassov [8], [9] introduced the intuitionistic fuzzy sets as a powerful extension of fuzzy set. Atanassov in his studies emphasized that in view of handling imprecision, vagueness or uncertainty in information both the degree of belonging and degree of non-belonging should be considered as two independent properties as these are not complement of each other. This concept of membership and non-membership was considered by Angelov [10] in optimization problem and gave intuitionistic fuzzy approach to solve optimization problems. Jana and Roy [11] studied the multi objective intuitionistic fuzzy linear programming problem and applied it to transportation problem. Luo [12] applied the inclusion degree of intuitionistic fuzzy set to multi criteria decision making problem. Further many workers such as Mahapatra et al., [13], Nachammai[14] and Nagoorgani [15] etc. have also studied linear programming problem under intuitionistic fuzzy environment. Recently Dubey et al., [16], [17] studied linear programming problem in intuitionistic fuzzy environment using intuitionistic fuzzy number and interval uncertainty in fuzzy numbers.

The motivation of the present study is to give computational algorithm for solving multi objective linear programming problem by intuitionistic fuzzy optimization approach. We also aim to study the impact of various type of membership and non-membership functions in such optimization process and thus have made comparative study of linear membership and non-membership function with that of nonlinear function for membership and nonmembership. The study has been organized in continuing 
sections as: Section two contains the preliminaries and basic principle of intuitionistic fuzzy optimization needed for developing algorithm. Section three contains two computational algorithms and the algorithm has been implemented on an illustration in section four and the result obtained has been placed in section five followed by references.

\section{PRELIMINARIES}

\section{A. Multi Objective Linear Programming Problem}

In general, a multi objective optimization problem with $p$ objectives, $q$ constraints and $n$ decision variables, is follows as

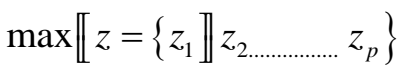

Such that $g_{j}(x) \leq 0, i=1.2 \ldots \ldots q$

$$
X_{i} \geq 0, i=1,2 \ldots \ldots \ldots \ldots n
$$

where $X=\left\{X_{1}, X_{2} \ldots \ldots . X_{n}\right\}$

\section{1) Complete solution}

$x^{0}$ is said to be a complete optimal solution for problem (1) if there exist $x^{0} \in X \quad$ such that $f_{k}\left(x^{0}\right) \geq f_{k}(x), \quad k=1,2, \ldots \ldots \ldots, p$, for all $x \in X$.

However, in general such complete optimal solutions that simultaneously maximize all of the multiple-objective function do not exist specially the objective functions are conflicting in nature. Thus instead of a complete optimal solution a solution concept, called Pareto optimality was introduced in multi-objective programming.

\section{2) Pareto-optimality}

$x^{0} \in X$ is said to be a Pareto optimal solution for (1) if there does not exist another $x \in x$ such that $f_{k}\left(x^{0}\right) \leq f_{k}(x)$ for all $p=1,2, \ldots \ldots ., p$ and $f_{j}\left(x^{0}\right)<f_{j}(x)$ for at least one $j \in\{1,2, \ldots \ldots, p\}$.

\section{B. Intuitionistic Fuzzy Sets}

Let $X$ be a non-empty set and $I=[0,1]$, then an IFS $\tilde{A}$ is defined as a set $\tilde{A}=\left\{<x, \mu_{\tilde{A}}(x), \nu_{\tilde{A}}(x)>: x \in X\right\}$ where $\mu_{\tilde{A}}: X \rightarrow I$ and $v_{\tilde{A}}: X \rightarrow I$ denotes the degree of belonging and the degree of non-belonging with $0 \leq \mu_{\tilde{A}}(x)+\nu_{\tilde{A}}(x) \leq 1$ for each $x \in X$.

Further, every fuzzy set $A$ on a non-empty set $X$ with membership function $\mu_{\tilde{A}}$ is obviously AN IF with $v_{\tilde{A}}(x)=1-\mu_{\tilde{A}}(x)$ and so IFS is a generalization of a fuzzy set.

Here union and intersection of two intuitionistic fuzzy sets are defined as

$$
\begin{aligned}
& \tilde{A} \cap \tilde{B}=\left\{\left[x, \min \left(\mu_{\tilde{A}}(x), \mu_{\tilde{B}}(x)\right),\right.\right. \\
& \left.\left.\max \left(v_{\tilde{A}}(x), v_{\tilde{B}}(x)\right)\right] \mid x \in X\right\}
\end{aligned}
$$

$$
\begin{aligned}
& \tilde{A} \cup \tilde{B}=\left\{\left[x, \max \left(\mu_{\tilde{A}}(x), \mu_{\tilde{B}}(x)\right),\right.\right. \\
& \left.\left.\min \left(v_{\tilde{A}}(x), v_{\tilde{B}}(x)\right)\right] \mid x \in X\right\} .
\end{aligned}
$$

Fuzzy Optimization Technique Max- min approach Zimmermann first used the max- min operator given by Bellman and Zadeh [18] to solve Multi Objective Linear Programming (MOLP) problems and considered the problem (1) as:

Find $X$

$$
\begin{array}{r}
\text { Such that } \quad Z_{k}(x) \tilde{\geq} g_{k}, k=1,2, \ldots \ldots, p . \\
g_{j}(x) \leq 0, \quad i=1.2 \ldots \ldots q \\
X \geq 0
\end{array}
$$

where $g_{k}, \forall x$, denote goals and all objective functions are assumed to be maximized. Here objective functions are considered as fuzzy constraints. To establish membership functions of objective functions, we could first obtain the table of positive ideal solution (PIS). Under the concept of min-operator, the feasible solution set is defined by interaction of the fuzzy objective set. This feasible solution set is then characterized by its membership $\mu_{D}(x)$ which is:

$$
\mu_{D}(x)=\min \left(\mu_{1}(x), \ldots \ldots \ldots, \mu_{k}(x)\right) \text {. }
$$

Further, a decision maker makes a decision with a maximum $\mu_{D}$ value in the feasible decision set. The decision solution can be obtained by solving the problem of maximize $\mu_{D}(x)$ subject to the given constraintsi.e.

$\operatorname{Max}\left[\min \mu_{k}(x)\right]$

Such that $g_{j}(x) \leq 0, i=1.2 \ldots \ldots q$

Now, if suppose $\alpha=\min _{k} \mu_{k}(x)$ be the overall satisfactory level of compromise, then we obtain the following equivalent model

$\operatorname{Max} \alpha$

Such that $\mu_{k}(x) \geq \alpha, \forall k$,

$$
g_{j}(x) \leq 0, \mathrm{j}=1.2 \ldots \ldots q X \geq 0
$$

\section{Intuitionistic Fuzzy Optimization Technique}

Consider the intuitionistic fuzzy optimization problem as generalization of the above problem a under taken by Angelov [3]

$$
\begin{array}{ll}
\min f_{i}(x), & i=1,2, \ldots \ldots p \\
g_{j}(x) \leq 0, & j=1,2, \ldots \ldots . q
\end{array}
$$

where, $x$ is decision variables, $f_{i}(x)$ denotes objective functions, $g_{j}(x)$ denotes the constraint functions, $p$ and $q$ denote the number of objective functions and constraints respectively.

The optimal solution of this problem must satisfy all constraints exactly. Thus an analogous fuzzy optimization model of the problem the degree of acceptance of objectives and constraints are maximized as:

$$
\text { min } f_{i}(x), \quad i=1,2, \ldots \ldots, p
$$




$$
g_{j}(x) \lesssim 0, \quad j=1,2, \ldots \ldots . q
$$

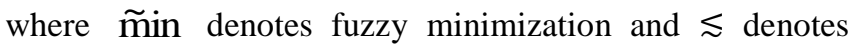
fuzzy inequality.

For solution of this system (5), Bellman and Zadeh [4] used fuzzy set maximize for the degree of membership of the objectives and constraints as

$$
\max \mu_{k}(x), \quad x \in X, k=1,2, \ldots ., p+q 0 \leq \mu_{k}(x) \leq 1
$$

where, $\mu_{k}(x)$ denotes the degree of satisfaction to respective fuzzy sets.

It is important to understand that in fuzzy set the degree of non-membership is complement of membership, hence maximization of membership function will automatically minimize the non-membership. But in intuitionistic fuzzy set degree of rejection is defined simultaneously with the degree of acceptance and both these degree are not complementary each other, hence IFS may give more general tool for describing this uncertainty based optimization model.

Thus, intuitionistic fuzzy optimization (IFO) model for problem(3) is given as

$$
\begin{gathered}
\max _{x}\left\{\mu_{k}(x)\right\}, \quad x \in X \quad k=1,2, \ldots \ldots \ldots, p+q \\
\min _{x}\left\{v_{k}(x)\right\}, \quad k=1,2, \ldots \ldots . p+q
\end{gathered}
$$

Such that

$$
\begin{array}{cl} 
& v_{k}(x) \geq 0, \quad k=1,2, \ldots \ldots . . p+q \\
\mu_{k}(x) \geq v_{k}(x), \quad & k=1,2, \ldots \ldots ., p+q \\
& \mu_{k}(x)+v_{k}(x) \leq 1, \quad k=1,2, \ldots \ldots ., p+q
\end{array}
$$

where, $\mu_{k}(x)$ denotes the degree of acceptance of $x$ to the $k^{\text {th }}$ IFS and $v_{k}(x)$ denotes the degree of rejection of $x$ from the $k^{\text {th }}$ IFS. These IFS include intuitionistic fuzzy objectives and constraints.

Now the decision set $\tilde{D}$ a conjunction of intuitionistic fuzzy objectives and constraints is defined as

$$
\tilde{F} \cap \tilde{C}=\left\{\begin{array}{l}
{\left[x, \min \left(\mu_{\tilde{F}}(x), \mu_{\tilde{C}}(x)\right),\right.} \\
\left.\max \left(v_{\tilde{F}}(x), v_{\tilde{C}}(x)\right)\right] \mid
\end{array} x \in X\right\}
$$

where, $\tilde{F}$ is integrated intuitionistic fuzzy objective and $\tilde{C}$ denotes integrated intuitionistic fuzzy constraints and is defined as:

$$
\begin{gathered}
\tilde{F}=\left\{\left[x, \mu_{\tilde{F}}(x), v_{\tilde{F}}(x)\right] \mid x \in X\right\}=\bigcap_{i=1}^{p} \tilde{F}^{(i)} \\
=\left\{\left[x, \min _{i=1}^{p} \mu_{i}^{f}(x), \max _{i=1}^{p} v_{i}^{f}(x)\right] \mid x \in X\right\} \\
\tilde{C}=\left\{\left[x, \mu_{\tilde{C}}(x), v_{\tilde{C}}(x)\right] \mid x \in X\right\}=\bigcap_{j=1}^{q} \tilde{C}^{(j)} \\
=\left\{\left[x, \min _{j=1}^{q} \mu_{j}^{g}(x), \max _{j=i}^{q} v_{j}^{g}(x)\right] \mid x \in X\right\}
\end{gathered}
$$

Further, the intuitionistic fuzzy decision set (IFDS) denoted as $\tilde{D}$ :

$$
\begin{gathered}
\tilde{D}=\tilde{F} \cap \tilde{C}=\left\{\left(x, \mu_{\tilde{D}}(x), v_{\tilde{D}}(x)\right) \mid x \in X\right\} \\
\mu_{\tilde{D}}(x)=\min \left[\mu_{\tilde{F}}(x), \mu_{\tilde{C}}(x)\right]=\min _{k=1}^{p+q} \mu_{k}(x) \\
v_{\tilde{D}}(x)=\max \left[v_{\tilde{F}}(x), v_{\tilde{C}}(x)\right]=\max _{k=1}^{p+q} v_{k}(x)
\end{gathered}
$$

where, $\mu_{\tilde{D}}(x)$ denotes the degree of acceptance of IFDS and $v_{\tilde{D}}(x)$ denotes the degree of rejection of IFDS.

Now for the feasible solution the degree of acceptance of IFDS is always less than or equal to the degree of acceptance of any objective and constraint and the degree of rejection of IFDS is always more than or equal to the degree of rejection of any objective and constraint, $i$, e.

$$
\begin{aligned}
& \mu_{\tilde{D}}(x) \leq \mu_{k}(x), \quad v_{\tilde{D}}(x) \geq v_{k}(x), \\
& \forall \quad k=1,2, \ldots \ldots, p+q
\end{aligned}
$$

Thus the above system can be transformed to the following system of inequalities:

$$
\begin{gathered}
\alpha \leq \mu_{k}(x), \quad k=1, \ldots ., p+q \\
\beta \geq v_{k}(x), \quad k=1, \ldots \ldots, p+q \\
\alpha+\beta \leq 1 \\
\alpha \geq \beta, \quad \beta \geq 0 \\
x \in X .
\end{gathered}
$$

where, $\alpha$ denotes the minimum acceptable degree of objective(s) and constraints, and $\beta$ denotes the maximum degree of rejection of objective(s) and constraints.

Now using the Intuitionistic fuzzy optimization the problem (1) is transformed to the linear programming problem given as:

$$
\begin{array}{cc}
\text { Maximize } & (\alpha-\beta) \\
\text { Subject to } & \alpha \leq \mu_{k}(x), \quad k=1, \ldots \ldots, p+q, \\
\beta \geq v_{k}(x), \quad & k=1, \ldots \ldots ., p+q, \\
\alpha+\beta \leq 1, \\
\alpha \geq \beta, \\
\beta \geq 0, \\
x \in X .
\end{array}
$$

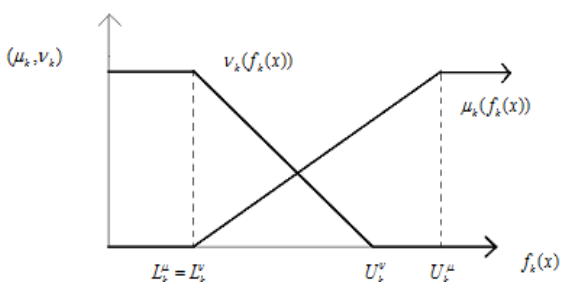

Fig. 1.The illustration of non-linear membership and non-linear nonmembership functions.

Now this linear programming problem can be easily solved by a simplex method to give solution of multiobjective linear programming problem (1) by intuitionistic 
fuzzy optimization approach.

Fig. 1 illustrates the linear membership and linear nonmembership functions.

\section{COMPUTATIONAL AlgORITHM}

\section{A. Algorithm I (Linear Membership Function)}

Step 1. Taking the first objective function from set of $k$ objectives of the problem and solve it as a single objective subject to the given constraints. Find value of objective functions and decision variables.

Step 2. From values of these decision variables compute values of remaining $(k-1)$ objectives.

Step 3. Repeat the Step 1 and Step 2 for remaining $(k-1)$ objective functions.

Step 4. Tabulate values of objective functions thus obtained from Step 1 and Step 2 and Step 3 to form to form a table known as PIS.

Step 5. From Step 4 obtain the lower bounds and upper bounds for each objective functions.

\begin{tabular}{|c|c|c|c|c|}
\hline \multirow[b]{2}{*}{$\begin{array}{l}\max f_{1} \\
\max f_{2} \\
\max f_{3} \\
: \\
: \\
\max f_{k}\end{array}$} & \multicolumn{3}{|l|}{$f_{1} f_{2} f_{3} f_{k}$} & $X$ \\
\hline & $\begin{array}{ll}f_{1}^{*} & f_{2}\left(X_{1}\right) \\
f_{1}\left(X_{2}\right) & f_{2}^{*} \\
f_{1}\left(X_{3}\right) & f_{2}\left(X_{3}\right) \\
: & \\
\vdots & \\
f_{1}\left(X_{k}\right) & f_{2}\left(X_{k}\right)\end{array}$ & $\begin{array}{c}f_{3}\left(X_{1}\right) \\
f_{3}\left(X_{2}\right) \\
f_{3}^{*} \\
\end{array}$ & $\begin{array}{cc}\ldots \ldots \ldots \ldots . . & f_{k}\left(X_{1}\right) \\
\ldots \ldots \ldots \ldots . . . & f_{k}\left(X_{2}\right) \\
\ldots \ldots \ldots \ldots . & f_{k}\left(X_{3}\right) \\
& \end{array}$ & $\begin{array}{l}X_{1} \\
X_{2} \\
X_{3} \\
: \\
: \\
X_{k}\end{array}$ \\
\hline & $f_{1}^{\prime} f_{2}^{\prime} f_{3}^{\prime} \ldots$ & & $f_{k}^{\prime}$ & \\
\hline
\end{tabular}

where $f_{k}^{*}$ and $f_{k}^{\prime}$ are the maximum, minimum values respectively.

Step 6. Set $U_{k}^{\mu}=\max \left(Z_{k}\left(X_{r}\right)\right) \quad$ and $L_{k}^{\mu}=\min \left(Z_{k}\left(X_{r}\right)\right), 1 \leq r \leq p$ for membership and for non-membership functions $U_{k}^{v}=U_{k}^{\mu}-\lambda\left(U_{k}^{\mu}-L_{k}^{\mu}\right) \quad$ and $L_{k}^{v}=L_{k}^{\mu}, 0<\lambda<1$.

Step 7. Use following linear membership function $\mu_{k}\left(f_{k}(x)\right)$ and non-membership function $v_{k}\left(f_{k}(x)\right)$ for each objective functions:

$$
\begin{gathered}
\mu_{k}\left(f_{k}(x)\right)=\left\{\begin{array}{ccc}
0 & \text { if } & f_{k}(x) \leq L_{k}^{\mu} \\
\frac{f_{k}(x)-L_{k}^{\mu}}{U_{k}^{\mu}-L_{k}^{\mu}} & \text { if } & L_{k}^{\mu} \leq f_{k}(x) \leq U_{k}^{\mu} \\
1 & \text { if } & f_{k}(x) \geq U_{k}^{\mu}
\end{array}\right. \\
v_{k}\left(f_{k}(x)\right)=\left\{\begin{array}{ccc}
0 & \text { if } & f_{k}(x) \geq U_{k}^{v} \\
\frac{U_{k}^{v}-f_{k}(x)}{U_{k}^{v}-L_{k}^{v}} & \text { if } & L_{k}^{v} \leq f_{k}(x) \leq U_{k}^{v} \\
1 & \text { if } & f_{k}(x) \leq L_{k}^{\mu}
\end{array}\right.
\end{gathered}
$$

Step 8. Now theintuitionistic fuzzy optimization method for MOLP problem (1) with linear membership and non membership functions gives a equivalent linear programming problem as :

Maximize $(\alpha-\beta)$

Subject to $\quad \alpha \leq \mu_{k}\left(f_{k}(x)\right)$,

$$
\begin{aligned}
& \beta \geq v_{k}\left(f_{k}(x)\right), \\
& \alpha+\beta \leq 1, \\
& \alpha \geq \beta, \\
& \quad \beta \geq 0, \\
& g_{j}(x) \leq b_{j}, x \geq 0, \\
& k=1,2, \ldots p ; \quad j=1,2, \ldots, q .
\end{aligned}
$$

Step 9. The above linear programming problem(14) can be easily solved by olve the above a simplex method.

\section{B. Algorithm II (Nonlinear Membership Function)}

Repeat steps 1 to step 6 and construct table of positive ideal solutions.

Step 7. Assume that solutions so for computed by algorithm follow hyperbolic function for membership and exponential for non-membership function given as

$$
\begin{gathered}
\mu_{k}\left(f_{k}(x)\right)=\left\{\begin{array}{l}
0, \quad f_{k}(x)<L_{k}^{\mu} \\
1-\operatorname{Exp}\left\{-\psi \frac{f_{k}(x)-L_{k}^{\mu}}{U_{k}^{\mu}-L_{k}^{\mu}}\right\} \quad, \quad L_{k}^{\mu} \leq f_{k}(x) \leq U_{k}^{\mu} \\
1, \quad f_{k}(x) \geq U_{k}^{\mu} \text { and } \psi \rightarrow \infty
\end{array}\right. \\
v_{k}\left(f_{k}(x)\right)= \begin{cases}1, & f_{k}(x) \leq L_{k}^{v} \\
\frac{1}{2}+\frac{1}{2} \tanh \left(\delta_{k} \cdot \frac{U_{k}^{v}+L_{k}^{v}}{2}-f_{k}(x)\right), \quad L_{k}^{v} \leq f_{k}(x) \leq U_{k}^{v} \\
0, & f_{k}(x) \geq U_{k}^{v}\end{cases}
\end{gathered}
$$

where $\psi, \delta_{k}$ are non zere parameters precribed by the decision maker.

Further, the intuitionistic fuzzy optimization technique for MOLP problem (1) with the exponential membership and hyperbolic non membership functions gives the following linear programming problem :

Maximize $(\alpha-\beta)$

Subject to $\alpha \leq \mu_{k}\left(f_{k}(x)\right)$,

$$
\begin{gathered}
1-\operatorname{Exp}\left\{-\psi \frac{f_{k}(x)-L_{k}^{\mu}}{U_{k}^{\mu}-L_{k}^{\mu}}\right\} \geq \alpha \\
\beta \geq v_{k}\left(f_{k}(x)\right), \\
\frac{1}{2}+\frac{1}{2} \tan h\left(\delta_{k} \frac{U_{k}^{v}+L_{k}^{v}}{2}-f_{k}(x)\right) \leq \beta, \\
\alpha+\beta \leq 1, \\
\alpha \geq \beta, \\
\beta \geq 0, \\
g_{j}(x) \leq b_{j}, x \geq 0 \\
k=1,2, \ldots . p ; \quad j=1,2, \ldots, q .
\end{gathered}
$$


For solution convenience the above problem (15) is transformed to

\section{Maximize $\gamma-\eta$}

Subject to $f_{k}(x)-\frac{\gamma\left(U_{k}^{\mu}-L_{k}^{\mu}\right)}{4} \geq L_{k}^{\mu}$, where $\gamma=-\log (1-\alpha$,

$$
f_{k}(x)-\frac{\eta}{\delta_{k}} \geq \frac{U_{k}^{v}+L_{k}^{v}}{2}
$$

where $\eta=-\tanh ^{-1}(2 \beta-1)$, and $\psi=4, \delta_{k}=\frac{6}{U_{k}^{v}-L_{k}^{v}}$

$$
\begin{gathered}
\gamma \geq \eta, \\
\gamma+\eta \leq 1, \\
\eta \geq 0, \\
g_{j}(x) \leq b_{j}, x \geq 0 \\
k=1,2, \ldots . p ; j=1,2, \ldots, q .
\end{gathered}
$$

Which can be easily solved by a simplex method.

\section{NUMERICAL ILLUSTRATION}

\section{A. Production Planning Problem}

Consider a park of six mechine types whose capacities are to be devoted to production of three products. A current capacity portfolio is available, measured in mechine hours per weak for each mechine capacity unit priced according to machine type.

Necessary data is summerized below Table II.

TABLE II: PHYSICAL PARAMETER VALUES

\begin{tabular}{|llllll|}
\hline Machine type & $\begin{array}{l}\text { Machi } \\
\text { hours }\end{array}$ & $\begin{array}{l}\text { Unit } \\
\text { price } \\
(\$ 100 \text { per } \\
\text { hour })\end{array}$ & $x_{1}$ & $x_{2}$ & $x_{3}$ \\
\hline $\begin{array}{l}\text { Milling } \\
\text { machine }\end{array}$ & 1400 & 0.75 & 12 & 17 & 0 \\
Lathe & 1000 & 0.60 & 3 & 9 & 8 \\
Grinder & 1750 & 0.35 & 10 & 13 & 15 \\
Jig saw & 1325 & 0.50 & 6 & 0 & 16 \\
Drill press & 900 & 1.15 & 0 & 12 & 7 \\
Band saw & 1075 & 0.65 & 9.5 & 9.5 & 4 \\
Total capacity cost $\$ 4658.75$ & & & \\
\hline
\end{tabular}

TABLE III: POSITIVE IDEAL SOLUTION

\begin{tabular}{|lllll|}
\hline & $f_{1}$ & $f_{2}$ & $f_{3}$ & $X$ \\
\hline $\operatorname{Max} f_{1}$ & 8041.14 & 10020.33 & 9319.25 & $X_{1}$ \\
$\operatorname{Max} f_{2}$ & 5452.63 & 10950.59 & 5903.00 & $X_{2}$ \\
$\operatorname{Max} f_{3}$ & 7983.60 & 10056.99 & 9355.90 & $X_{3}$ \\
\hline
\end{tabular}

Let $x_{1}, x_{2}, x_{3}$ denote three products, then the complete mathematical formulation of the above mentioned problem as a Multi objective Linear Programming (MOLP) problem is given as:

$$
\begin{aligned}
& \operatorname{Max} f_{1}(x)=50 x_{1}+100 x_{2}+17.5 x_{3} \text { (profit) } \\
& \operatorname{Max} f_{2}(x)=92 x_{1}+75 x_{2}+50 x_{3} \quad \text { (quality) }
\end{aligned}
$$

Ma $f_{3}(x)=25 x_{1}+100 x_{2}+75 x_{3}$ (worker satifaction) Subject to the constraints

$$
\begin{gathered}
12 x_{1}+17 x_{2} \leq 1400 \\
3 x_{1}+9 x_{2}+8 x_{3} \leq 1000 \\
10 x_{1}+13 x_{2}+15 x_{3} \leq 1750 \\
6 x_{1}+16 x_{3} \leq 1325 \\
x_{1}, \quad x_{2}, \quad x_{3} \geq 0 .
\end{gathered}
$$

\begin{tabular}{|c|c|c|c|c|c|}
\hline \multirow[b]{2}{*}{$\lambda$} & \multicolumn{5}{|c|}{$\begin{array}{l}\text { Intuitionistic fuzzy optimization Technique when } \\
\text { membership and Non- memberships are linear. }\end{array}$} \\
\hline & $x_{1}$ & $x_{2}$ & $x_{3}$ & $\alpha$ & $\beta$ \\
\hline .1 & 65.2571 & 26.9187 & 49.8324 & .5899 & .4101 \\
\hline .2 & 58.4833 & 34.5907 & 47.6992 & .8525 & .1475 \\
\hline .3 & 65.2600 & 26.9155 & 49.8333 & .7583 & .2417 \\
\hline .4 & 65.2585 & 26.9172 & 49.8328 & .8847 & .1153 \\
\hline .5 & 66.1947 & 25.8441 & 49.2978 & 1.000 & .0000 \\
\hline .6 & 71.1362 & 22.6184 & 44.8504 & 1.000 & .0000 \\
\hline .7 & 71.7199 & 25.7841 & 35.6084 & 1.000 & .0000 \\
\hline .8 & 75.3355 & 14.2823 & 45.3258 & 1.000 & .0000 \\
\hline .9 & 82.1131 & 9.12270 & 46.1075 & 1.000 & .0000 \\
\hline & \multicolumn{5}{|c|}{$\begin{array}{l}\text { Intuitionistic fuzzy optimization Technique when } \\
\text { membership and Non- memberships are Non-linear }\end{array}$} \\
\hline$\lambda$ & $x_{1}$ & $x_{2}$ & $x_{3}$ & $\alpha$ & $\beta$ \\
\hline .1 & 49.8906 & 47.1360 & 42.5550 & .6321 & .3345 \\
\hline .2 & 64.6968 & 36.6846 & 41.7421 & .6321 & .0073 \\
\hline .3 & 62.1896 & 38.0097 & 41.8452 & .6321 & .0009 \\
\hline .4 & 62.8180 & 38.0109 & 41.5300 & .6321 & .0001 \\
\hline .5 & 62.8157 & 38.0125 & 41.8454 & .6321 & .0000 \\
\hline .6 & 62.8163 & 38.0120 & 41.8454 & .6321 & .0000 \\
\hline .7 & 59.7690 & 40.1631 & 42.0127 & .6321 & .0000 \\
\hline .8 & 62.8265 & 38.0048 & 41.8448 & .6321 & .0000 \\
\hline .9 & 62.8207 & 38.0087 & 41.8451 & .6321 & .0000 \\
\hline
\end{tabular}

Solution of the above problem is considered by the algorithm I and algorithm II mentioned in previous sections. For illustration of the procedures some of steps are shown as

Step 1. Solve a linear programming problem taking one objective

Maximize $f_{1}=50 x_{1}+100 x_{2}+17.5 x_{3}$

Subject to the constraints

$$
\begin{array}{r}
12 x_{1}+17 x_{2} \leq 1400 \\
3 x_{1}+9 x_{2}+8 x_{3} \leq 1000 \\
10 x_{1}+13 x_{2}+15 x_{3} \leq 1750 \\
6 x_{1}+16 x_{3} \leq 1325 \\
12 x_{2}+7 x_{3} \leq 900 \\
9.5 x_{1}+9.5 x_{2}+4 x_{3} \leq 1075 \\
x_{1}, \quad x_{2}, \quad x_{3} \geq 0 .
\end{array}
$$

TABLE IV: VALUES OF OPTIMAL DECISION VECTORS

Optimal solution to this crisp linear programming problem is

$$
\begin{gathered}
x_{1}=44.93, \quad x_{2}=50.63, x_{3}=41.77, \\
\left(f_{1}\right)_{1}=8041.14
\end{gathered}
$$

Step 2. Withthese decision variables, computed values of other remaining objective functions are:

$$
\left(f_{2}\right)_{1}=10020.33
$$




$$
\left(f_{3}\right)_{1}=9319.25
$$

Step 3. Step 1 and Step 2 are repeated for other objective functions $f_{2}, f_{3}$.

Step 4. The Positive Ideal Solution (PIS) obtained are placed in Table III.

Step 5. Applying the solution algorithm I and algorithm II , the solutions of the mentioned MOLP are obtained. The problem is solved by linear membership and non membership and is also solved by nonlinear membership and nonmembership functionusing various values of $\lambda \alpha \beta$ the solutions thus obtained are placed in Table IV to have insight in the solution process. The feasibility of solutions in view of various satisfaction levels are depicted in the Table V.

TABLE V: VALUES OF OPTIMAL OBJECTIVE FUNCTIONS

\begin{tabular}{|c|c|c|c|c|}
\hline \multirow[b]{2}{*}{$\lambda$} & \multicolumn{4}{|c|}{$\begin{array}{l}\text { Intuitionistic fuzzy optimization Technique when } \\
\text { membership and Non- memberships are linear. }\end{array}$} \\
\hline & $\max f_{1}$ & $\max f_{2}$ & $\max f_{3}$ & Total \\
\hline 0.1 & 6826.7920 & 10514.1757 & 8060.7275 & 25401.6952 \\
\hline 0.2 & 7217.9710 & 10359.7261 & 8498.5925 & 26076.2896 \\
\hline 0.3 & 6826.6328 & 10514.2475 & 8060.5475 & 25401.4278 \\
\hline 0.4 & 6826.7190 & 10514.2120 & 8060.6425 & 25401.5735 \\
\hline 0.5 & 6756.8565 & 10493.1099 & 7936.6125 & 25186.5789 \\
\hline 0.6 & 6603.5320 & 10483.4304 & 7404.0250 & 24490.9874 \\
\hline 0.7 & 6787.5520 & 10312.4583 & 7041.0375 & 24142.0478 \\
\hline 0.8 & 5988.2065 & 10268.3285 & 6711.0525 & 22967.5875 \\
\hline 0.9 & 5824.8063 & 10543.9827 & 6423.1600 & 22791.9490 \\
\hline \multirow[b]{2}{*}{$\lambda$} & \multicolumn{4}{|c|}{$\begin{array}{l}\text { Intuitionistic fuzzy optimization Technique when } \\
\text { membership and Non- memberships are non-linear. }\end{array}$} \\
\hline & $\max f_{1}$ & $\max f_{2}$ & $\max f_{3}$ & Total \\
\hline 0.1 & 7952.8425 & 10252.8852 & 9152.4900 & 27358.2177 \\
\hline 0.2 & 7633.7868 & 10790.5556 & 8416.5375 & 26840.8793 \\
\hline 0.3 & 7642.7410 & 10664.4307 & 8494.1000 & 26801.2717 \\
\hline 0.4 & 7668.7650 & 10706.5735 & 8486.2900 & 26861.6285 \\
\hline 0.5 & 7674.3295 & 10722.2519 & 8510.0475 & 26906.6289 \\
\hline 0.6 & 7674.3095 & 10722.2696 & 8510.0125 & 26906.5916 \\
\hline 0.7 & 7737.9823 & 10611.6155 & 8661.4875 & 27011.0853 \\
\hline 0.8 & 7674.0890 & 10722.6380 & 8509.5025 & 26906.2295 \\
\hline 0.9 & 7674.1942 & 10722.4119 & 8509.7700 & 26906.3761 \\
\hline
\end{tabular}

TABLE VI: COMPARISON OF OPTIMAL SOLUTIONS OBTAINED BY VARIOUS METHODS

\begin{tabular}{|c|c|c|c|}
\hline $\begin{array}{l}\text { Decision } \\
\text { variables \& } \\
\text { objective } \\
\text { functions }\end{array}$ & $\begin{array}{l}\text { Best Solution } \\
\text { obtained by } \\
\text { fuzzy } \\
\text { optimization } \\
\text { method with } \\
\text { level of satis } \\
\text { faction } \\
\alpha=0.5309\end{array}$ & $\begin{array}{l}\text { Best } \\
\text { Solution } \\
\text { obtained by } \\
\text { proposed } \\
\text { intutionistic } \\
\text { fuzzy } \\
\text { optimization } \\
\text { alogirthm I }\end{array}$ & $\begin{array}{l}\text { Best } \\
\text { Solution } \\
\text { obtained by } \\
\text { proposed } \\
\text { intutionistic } \\
\text { fuzzy } \\
\text { optimization } \\
\text { alogirthm II }\end{array}$ \\
\hline $\mathrm{X}_{1}$ & 65.2571 & 58.4833 & 49.8906 \\
\hline $\mathrm{x}_{2}$ & 26.9187 & 34.5907 & 47.1360 \\
\hline $\mathrm{X}_{3}$ & 49.8324 & 47.6992 & 42.5550 \\
\hline $\mathrm{f}_{1}$ & 6826.7920 & 7217.9710 & 7952.8425 \\
\hline$f_{2}$ & 10514.1757 & 10359.7261 & 10252.8852 \\
\hline $\mathrm{f}_{3}$ & 8060.7275 & 8498.5925 & 9152.4900 \\
\hline $\begin{array}{l}\text { Sum of } \\
\text { objectives }\end{array}$ & 25401.6952 & 26076.2896 & 27358.2177 \\
\hline
\end{tabular}

\section{CONCLUSions}

In view of comparing the intuitionistic fuzzy optimization with fuzzy optimization method, we also obtained the solution of the undertaken numerical problem by fuzzy optimization method given by Zimmermann [17] and took the best result obtained for comparison with present study. We considered the best solution obtained by the developed two algorithms and are placed in Table VI for comparison with each other and also to compare with the results obtained by fuzzy optimization method.

The objective of the present study is to give the effective algorithm for intuitionistic fuzzy optimization method for getting optimal solutions to a multi objective linear programming problem. The merit of the method lies with fact that it gives a set of solutions with various level of satisfaction to the decision makers. The decision makers may choose a suitable optimal solution according to the demand of the actual situation. Further the comparisons of results obtained for the undertaken problem clearly show the superiority of intuitionistic fuzzy optimization over fuzzy optimization. The results thus obtained also reveal that intuitionistic fuzzy optimization by proposed algorithm II using nonlinear membership and nonlinear non membership give a better result than intuitionistic fuzzy optimization algorithm $I$ using linear membership function and linear non membership function.

\section{ACKNOWLEDGEMENTS}

The authers are thankful to University Grants Commission (U.G.C) NEW DELHI, INDIA, for financial support for research work. Authors also thank to DSTCentre for Interdisciplinary Mathematical Sciences, BHU for providing their support.

\section{REFERENCES}

[1] H. J. Zimmermann, "Fuzzy programming and linear programming with several objective functions," Fuzzy Sets and Systems, vol. 1, pp. 45-55, 1978.

[2] H. J. Zimmermann, "Fuzzy mathematical programming," Comput. Oper. Res.,vol. 10, pp.1-10, 1984.

[3] L.A. Zadeh, "Fuzzy Sets," Information and control, vol. 8, pp. 338353, 1965.

[4] H. Tanaka and K. Asai, "Fuzzy linear programming problems with fuzzy numbers," Fuzzy Sets and Systems, vol.139, pp. 1-10, 1984.

[5] M. Luhandjula, "Fuzzy optimization: an appraisal," Fuzzy Sets and Systems, vol. 30, pp. 257-288,1988.

[6] M. Sakawa and H. Yano, "An iteractive fuzzy satisfying method of multi objective nonlinear programming problems with fuzzy parameters," Fuzzy sets and Systems, vol. 30, pp. 221-238, 1989.

[7] N. V.Sahindis,"Optimization under uncertainty: state-of-the- art and opportunities," Computers and Chemical Engineering, vol. 28, pp. 971-983, 2004.

[8] K. T. Atanassov, "Intuitionistic fuzzy sets," Fuzzy Sets and Systems, vol. 20, pp. 87-96, 1986.

[9] K.T. Atanassov, "Interval valued intuitionistic fuzzy sets," Fuzzy Sets and Systems, vol. 31, pp. 343-349, 1989.

[10] P. P. Angelov, "Optimization in an intuitionistic fuzzy environment," Fuzzy Sets and Systems, vol. 86, pp. 299-306, 1997.

[11] B. Jana and T. K. Roy, "Multi objective intuitionistic fuzzy linear programming and its application in transportation model," NIFS vol. 13, no. 1, pp. 1-18, 2007.

[12] Y. Luo and C. Yu, "An fuzzy optimization method for multi criteria decision making problem based on the inclusion degrees of intuitionistic fuzzy set," Journal of Information and Computing Science, vol. 3, no. 2, pp. 146-152, 2008.

[13] G. S. Mahapatra, M. Mitra, and T. K. Roy, "Intuitionistic fuzzy multiobjective mathematical programming on reliability optimization model," International Journal of Fuzzy Systems, vol. 12, no. 3, pp. 259-266, 2010.

[14] A. L. Nachammai and P Thangaraj, "Solving intuitionistic fuzzy linear programming problem by using similarity measures," European Journal of Scientific Research, vol. 72, no. 2, pp. 204-210, 2012. 
[15] P. K. Nagoorgani, "A new approach on solving Intuitionistic fuzzy linear programming problem," Applied Mathematical Sciences, vol. 6 no. 70, pp. 3467-3474, 2012.

[16] D. Dubey and A. Mehra, "Linear programming with Triangular Intuitionistic Fuzzy Number," Eusflat-Lfa2011, Advances in Intelligent Systems Research, Atlantis Press, vol. 1, no. 1, pp. 563$569,2011$.

[17] D. Dubey, S. Chandra, and A. Mehra, "Fuzzy linear programming under interval uncertainty based on IFS representation," Fuzzy Sets and Systems, vol. 188, no. 1, pp. 68-87, 2012.

[18] R. E. Bellman and L. A. Zadeh, "Decision making in a fuzzy environment," Management Science, vol. 17, pp. B141-B164, 1970.

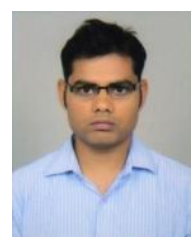

Shailendra Kumar Bharati was born in 1987. He received his M.Sc. degree in mathematics in 2010 from the Department of Mathematics, Banaras Hindu University, India. Currently, he is working as a senior research fellow and persuing Ph.D. degree in mathematics from Banaras Hindu University. His studies are mainly focused on fuzzy sets, intuitionistic fuzzy sets and its application in operations research.

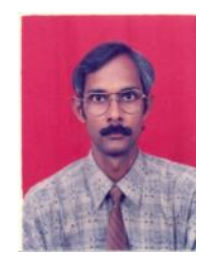

Shiva Raj Singh was born in Mirzapur, India in 1960 and received his M.Sc. and Ph.D. degree in mathematics from Banaras Hindu University, India in 1980 and 1985 respectively. Currently, he is a professor with the Department of Mathematics at Banaras Hindu University, India. Varanasi, India. His area of interest is soft computing: Fuzzy and neuoro fuzzy computing in decision making and forecasting problems. His area of expertise includes fuzzy time series forecasting, fuzzy optimization, multi objective programming and intuitionistic fuzzy optimization. 